\title{
Erwärmung von Materialien und Werkstoffen durch Anwendung von Mikrowellentechnologien
}

\author{
Lutz Hartmann, Tiberiu-Mihai Tatai, Mario Kaiser und Christian Dreyer*
}

\section{Zusammenfassung}

Die Erwärmung unterschiedlicher Materialien im Kontext verschiedener Anwendungen kann anhand der Bestrahlung mit Mikrowellen in geeignet konstruierten Öfen erfolgen. Der vorliegende Beitrag beginnt mit einer Vorstellung der mikroskopischen Mechanismen der Erwärmung von Materie mittels Mikrowellen sowie einem Abriss ihrer theoretischen Beschreibung. Im Folgenden werden die breitbandige dielektrische Spektroskopie und die Simulation elektromagnetischer Felder als Hilfsmittel eingeführt, die Effizienz des mikrowellenbasierten Heizens für bestimmte Materialien vorab einschätzen zu können. Nach der Vorstellung zweier unterschiedlicher Mikrowellenanlagen, namentlich eines Kammer- und eines Durchlaufmikrowellenofens, wird die thermische Behandlung unterschiedlicher Materialien mittels Mikrowellen an drei Beispielen illustriert.

\begin{abstract}
The heating of various materials in the context of different applications can be achieved by irradiation with microwaves in properly constructed microwave ovens. This article starts with an introduction to the microscopic mechanisms of heating of matter followed by a survey of their theoretical description. Both, broadband dielectric spectroscopy and the simulation of electromagnetic fields will be presented as convenient tools to estimate the efficiency of microwave-based heating of certain materials prior to any experiment. In addition to an introduction of two different microwave ovens, namely a chamber oven and a throughput oven, the thermal treatment of different materials is illustrated by three examples.
\end{abstract}

\section{Einführung}

Neben der konventionellen thermischen Behandlung von Materialien, wie etwa das Härten von faserverstärkten Kunststoffen in Öfen, haben in den letzten Jahren alternative Heizverfahren zunehmend an Bedeutung gewonnen. Eine besondere Rolle nimmt hierbei die Erwärmung von Materialien mittels Mikrowellen ein, die auf der Umwandlung elektromagnetischer Feldenergie in Wärme beruht. Die mikroskopischen Mechanismen, die diesem Erwärmungsprozess zugrunde liegen, sind die dissipative Reorientierung permanenter Dipolmomente und die dissipative Bewegung von Ladungsträgern in der Probe unter dem Einfluss des äußeren elektromagnetischen Feldes. Die Effizienz dieser Energiewandlung ist durch die komplexe dielektrische Funktion und ihre Temperatur- bzw. Frequenzabhängigkeit bestimmt, die in einem ausreichend weiten Temperatur- und Frequenzfenster mittels der breitbandigen dielektrischen Spektroskopie gemessen werden kann. Mit diesen Messungen eröffnet sich auch die Möglichkeit, die elektromagnetischen Felder und, daraus abgeleitet, die Verteilungen der Leistungsverlustdichte in Mikrowellenöfen zu simulieren. Im vorliegenden Beitrag wird nach einer Darlegung der Grundlagen des Heizens mittels Mikrowellen das Zusammenspiel zwischen dielektrischen Messungen und Simulationen diskutiert. Darüber hinaus werden praktische Beispiele für die Erwärmung unterschiedlicher Materialien mittels Mikrowellen angegeben und diskutiert.
2. Erwärmung von Materialien mittels Mikrowellen

Es werden im Folgenden für die beiden maßgeblichen Mechanismen der Erwärmung von Materialien mittels Mikrowellen die jeweils grundlegenden Zusammenhänge dargelegt. Dabei handelt es sich um das für Dielektrika relevante sog. dielektrische Heizen, das aufgrund einer materialabhängigen Eindringtiefe als volumetrisches Heizen angesehen werden kann. Im Falle elektrisch leitender Materialien ist hingegen das sog. Wirbelstrom-Heizen relevant, das aufgrund vergleichsweise geringer Eindringtiefen als ein Heizprozess nur oberflächennaher Bereiche anzusehen ist. Für eine ausführliche Ableitung und Diskussion beider Mechanismen sei auf die Literatur verwiesen (Feher 2009, Imenokhoyev et al. 2012, Meredith 2007, Perelaer et al. 2006, Zhang et al. 2017). 


\subsection{Dielektrisches Heizen}

Das dielektrische Heizen basiert auf der Absorption elektromagnetischer Wellen in Dielektrika, die permanente molekulare Dipolmomente aufweisen. Deren durch das äußere elektromagnetische Feld induzierte Fluktuationen sind dissipativ infolge mikroskopischer Reibung, so dass sich ein äußeres Mikrowellenfeld in eine Erwärmung des Materials übersetzt. Im Unterschied zu konventionellen Öfen, bei denen der Wärmetransport stets durch die Materialoberfläche erfolgt, findet beim dielektrischen Heizen der Wärmeeintrag in das Material im Wesentlichen innerhalb einer Eindringtiefe $d_{p}$ statt, für die gilt: $d_{p} \sim \frac{\sqrt{\varepsilon^{\prime}}}{f \varepsilon^{\prime \prime}}$ (Meredith 2007). Dabei sind $\varepsilon$ sowie $\varepsilon$ " der frequenz- und temperaturabhängige Real- bzw. Imaginärteil der komplexen dielektrischen Funktion bzw. Permittivität $\varepsilon^{*}(f, T)=\varepsilon^{\prime}(f, T)-i \varepsilon$ " $(f, T)$ und $f$ die Frequenz des elektromagnetischen Feldes (bei Mikrowellenöfen typischerweise 2,45 GHz). Für die innerhalb der Eindringtiefe dissipierte Leistungsdichte $p$ gilt $p \sim f \varepsilon^{\prime \prime} E_{i}^{2}$ (Imenokhoyev et al. 2012), wobei $E_{i}$ die Feldstärke im Inneren des Materials ist. Aus den Formeln zur Eindringtiefe und zur Leistungsverlustdichte ergibt sich unmittelbar die Bedeutung der in Abschnitt 3.1 dargestellten Messung der komplexen dielektrischen Funktion $\varepsilon^{*}(f, T)$ im relevanten Frequenz- bzw. Temperaturbereich, um die Effizienz der Erwärmung von Dielektrika vorab abschätzen oder simulieren zu können.

Die hauptsächlichen Vorteile des dielektrischen Heizens gegenüber konventionellen Heizprozessen sind der direkte Energieeintrag in das Material und die daraus resultierende kürzere Aufheizzeit. Dem steht die Beschränkung auf Materialien gegenüber, die aufgrund ihrer dielektrischen Permittivität eine effektive Wandlung von elektromagnetischen Wellen in Wärme zulassen. Dieser Punkt lässt sich bei der Härtung von Reaktivharzen und daraus hergestellten Composites durch die Zugabe von Partikeln, die ihrerseits die dielektrischen Kennwerte modifizieren, entscheidend verbessern (Akhtar et al. 2009, Link 2011, Link 2015).

\subsection{Wirbelstrom-Heizen}

Wirbelströme werden in einem elektrischen Leiter mit einem elektrischen Widerstand in einem sich zeitlich ändernden Magnetfeld induziert und führen in der Folge zu einer Erwärmung des Leiters. Die Heiz-Leistung des Wirbelstromheizens ist gegeben zu $P \sim I^{2} \sqrt{\mu f / \sigma}$ mit der magnetischen Permeabilität $\mu$, der elektrischen Leitfähigkeit $\sigma$ des Leiters und der Frequen $z$ des äußeren elektromagnetischen Feldes (Zhang et al. 2017). Die Wirbelströme erzeugen wiederum ein Magnetfeld, das gemäß der Lenzschen Regel seiner Ursache entgegenwirkt und zu einer Verdrängung des Stromflusses aus der Leitermitte führt (Skin-Effekt). Die daraus resultierende sog. Leitschichtdicke $\delta$ ist gegeben zu $\delta=1 / \sqrt{\pi \mu \sigma f}$ (Zhang et al. 2017). Diese Dicke nimmt mit steigender Frequenz ab und beträgt 1 bis $2 \mu \mathrm{m}$ bei Metallen und Frequenzen $f \approx$ $1 \mathrm{GHz}$ (Perelaer et al. 2006).

Aufgrund seiner intrinsischen Eigenschaften, insbesondere der Begrenzung auf elektrisch leitende Materialien und der Beschränkung auf die Erwärmung nur oberflächennaher Bereiche, deren Dicke durch die Eindringtiefe des Skin-Effektes gegeben ist, erscheint das Wirbelstrom-Heizen im Vergleich zum dielektrischen Heizen für praktische Anwendungen weniger relevant. Gegenwärtig untersucht wird jedoch zum einen das mikrowellen-basierte Sintern gedruckter leitfähiger Strukturen zur Einstellung ihrer endgültigen Leitfähigkeit, wobei der mikrowellenbasierte Ansatz insbesondere bei temperaturempfindlichen und gleichzeitig mikrowellentransparenten Substraten herkömmlichen Verfahren überlegen ist (Perelaer et al. 2006, Perelaer et al. 2009). Bei der Mikrowellenbestrahlung von Composites - mit leitfähigen Carbonfasern als Verstärkungsmaterial - ist zum anderen eine vorsichtige Prozessführung zwingend erforderlich, um ein zu starkes Erwärmen des Materials infolge des Wirbelstrom-Heizens der Carbonfasern zu verhindern.
3. Breitbandige dielektrische Spektroskopie und numerische Simulation elektromagnetischer Felder

Aus Abschnitt 2 wird ersichtlich, dass die Kenntnis der komplexen dielektrischen Funktion $\varepsilon^{*}(f, T)$ wie auch der Leitfähigkeit in ihrer Frequenz- und Temperaturabhängigkeit zwingend erforderlich ist, um die o. g. Mechanismen des Heizens mittels Mikrowellen in ihrer Wirkung abschätzen zu können bzw. zu simulieren. Beide Aspekte, die Messung der dielektrischen Kennwerte und die Simulation elektromagnetischer Felder in den Mikrowellenöfen, werden im Folgenden dargelegt.

\subsection{Breitbandige dielektrische Spektroskopie}

\subsubsection{Grundlagen}

Gegenstand der dielektrischen Spektroskopie ist die Untersuchung der Reaktion von Materie auf ein äußeres elektrisches Feld $\boldsymbol{E}$ (Kremer \& Schönhals 2002). Ausgehend von den Maxwellschen Gleichungen lässt sich das elektrische Feld $\boldsymbol{E}$ mit der dielektrischen Verschiebung $\boldsymbol{D}$ im Dielektrikum verknüpfen über $\boldsymbol{D}=\varepsilon_{0} \boldsymbol{E}+\boldsymbol{P}$, wobei $\varepsilon_{0}=8,8510^{-12} \mathrm{AsV}^{-1} \mathrm{~m}^{-1}$ die Permittivität des Vakuums ist. Die Polarisation $\boldsymbol{P}$ ist die Summe aller $N$ mikroskopischen Dipolmomente $p_{i}$, die sich in einem Volumen $V$ befinden: $\boldsymbol{P}=1 / \mathrm{V} \sum_{\mathrm{i}=1}^{\mathrm{N}} \boldsymbol{p}_{\mathrm{i}}$, wobei die Dipolmomente $p_{i}$ entweder permanent sind oder durch das elektrische Feld $\boldsymbol{E}$ induziert werden. Schließlich lässt sich die dielektrische Funktion bzw. Permittivität $\varepsilon^{*}(f, T)$ als Verknüpfung zwischen elektrischer Feldstärke $\boldsymbol{E}$ und dielektrischer Verschiebung $\boldsymbol{D}$ einführen: $\boldsymbol{D}=\varepsilon_{0} \boldsymbol{E}+\boldsymbol{P}=\varepsilon^{*}(f, T) \varepsilon_{0} \boldsymbol{E}$.

Wird die stationäre Antwort eines Dielektrikums auf ein harmonisches elektrisches Feld $\boldsymbol{E}(t)=\boldsymbol{E}_{0} \mathrm{e}^{-i \omega t}$ mit der Kreisfrequenz $\omega=2 \pi f$ betrachtet, so folgt die Antwort des Dielektrikums dem äußeren Feld mit zeitlicher Verzögerung und es kommt zu einer Phasenverschiebung zwischen elektrischer Feldstärke $\boldsymbol{E}(t)$ und dielektrischer Verschiebung $\boldsymbol{D}(t)$. Die dielektrische Funktion $\varepsilon^{*}(f, T)$ wird dann zweckmäßig als komplexe Größe definiert: $\varepsilon^{*}(f, T)=\varepsilon^{\prime}(f, T)-i \varepsilon$ " $(f, T)$. Sie wird durch die Messung der Kapazität eines leeren bzw. mit Dielektrikum 
gefüllten Kondensators experimentell zugänglich. Infolge eines äußeren elektrischen Feldes werden in einem Dielektrikum durch die Verschiebung von Ladungsschwerpunkten gegeneinander Dipolmomente erzeugt. Diese tragen gemäß den folgenden Mechanismen zur Verschiebungspolarisation bei:

- Elektronische Polarisation: Die Ladungsschwerpunkte von Elektronenhülle und Kern verschieben sich gegeneinander mit einer Zeitkonstante von $\approx 10^{-14} \mathrm{~s}$.

- lonische Polarisation: Die Verschiebung von Ionen in einem Kristallgitter ist aufgrund der größeren Teilchenmasse um etwa zwei Größenordnungen langsamer.

- Maxwell-Wagner- oder Elektrodenpolarisation: Bewegliche Ladungsträger in der Probe driften aufgrund eines äußeren elektrischen Feldes. Diese Drift wird durch innere Oberflächen oder die Elektroden blockiert und es tritt eine zusätzliche Polarisation auf.

Der bisher diskutierte Prozess der dielektrischen Relaxation setzte ideal isolierende Dielektrika voraus. Er ist oft durch eine elektrische Leitfähigkeit aufgrund beweglicher Ladungsträger in einer realen Probe überlagert. Diese Leitfähigkeit führt zu einer Stromdichte $\boldsymbol{j}$ im Material, für die sich schreiben lässt $\boldsymbol{j}=\sigma^{*}(f, T) \boldsymbol{E}$ mit $\sigma^{*}(f, T)=\sigma^{\prime}(f, T)-i \sigma^{\prime \prime}(f, T)$ als komplexer Leitfähigkeit (Ohmsches Gesetz). Im Falle einer rein ohmschen Gleichstromleitfähigkeit $\sigma_{0}$ erhält man einen Beitrag zum dielektrischen Verlust $\varepsilon$ " von $\sigma_{0} \omega^{-s} a \varepsilon_{0}$. Hierbei ist $s$ ein Exponent mit $0<s<1$ und $a$ ist ein Korrekturfaktor.

Eine Beschreibung des vollständigen Spektrums $\varepsilon^{*}(f, T)$ erfolgt zumeist mittels der verallgemeinerten Relaxationsfunktion von Havriliak und Negami (Kremer et al. 2002), die in der Frequenzdomäne gegeben ist zu

$$
\varepsilon_{H N}^{*}(\omega)=\varepsilon_{\infty}+\frac{i \sigma_{0} a}{\varepsilon_{0} \omega^{s}}+\frac{\Delta \varepsilon}{\left(1+\left(i \omega \tau_{H N}\right)^{\alpha}\right)^{\gamma}}
$$

mit $\Delta \varepsilon$ als Relaxationsstärke und $t_{H N}$ als Relaxationszeit. Als Zusammenfassung dieses Abschnittes zeigt Abb. 1 exemplarisch die zeitliche Entwicklung des Real- bzw. Imaginärteils der dielektrischen Funktion während der thermischen Härtung eines Phenolharz-Prepregs samt Anpassungen der Havriliak-Negami-Funktion an die Daten.
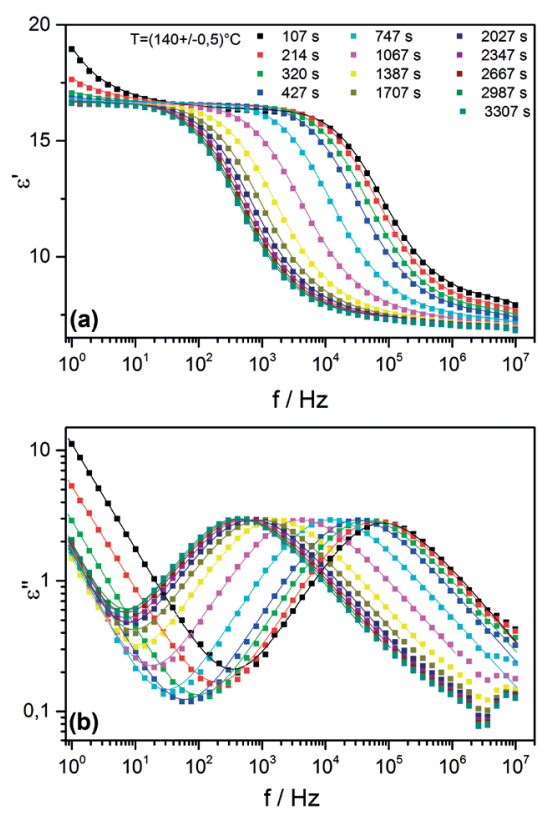

Abb. 1) Dielektrische Spektren ((a) Realteil $\varepsilon$; (b) Imaginärteil $\varepsilon$ ") gemessen während der isothermen Härtung eines Phenolharz/Glasfaser-Prepregs: Die Symbole repräsentieren Messwerte, die Linien stellen Anpassungen der HN-Funktion an die Daten dar. Alle Spektren sind durch einen Relaxationsprozess und einen Leitfähigkeitsbeitrag bei niedrigen Frequenzen charakterisiert.

Ein weiteres Beispiel ist in Abb. 2 angegeben: Hier wird gezeigt, wie sich die effektiven dielektrischen Kennwerte eines kommerziellen CyanatesterReinharzes (L 10) durch die Zugabe von Partikeln, in diesem Falle Siliziumcarbid $\mathrm{SiC}$, in Konzentrationen von 1 Ma-\% und 3 Ma-\% modifizieren lassen. Die Daten gelten für eine Frequenz des elektrischen Feldes von $1 \mathrm{GHz}$ und können daher als relevant für die Bestrahlung mit Mikrowellen bei 2,45 $\mathrm{GHz}$ angesehen werden.
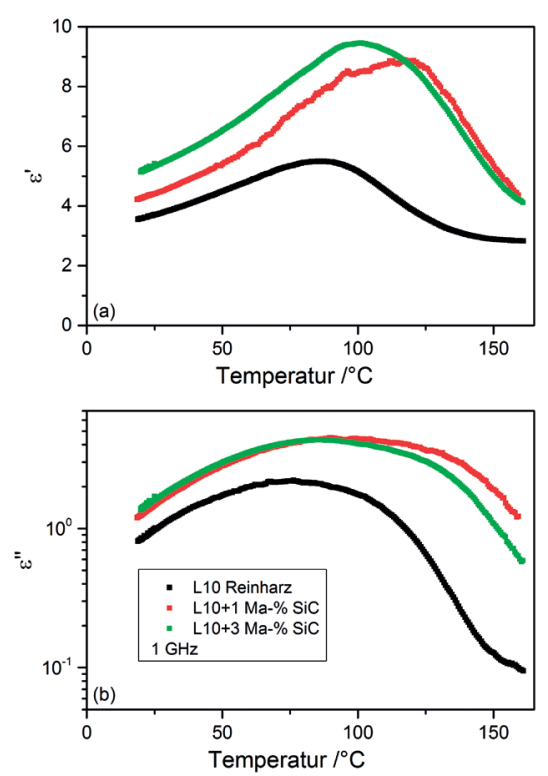

Abb. 2) Dielektrische Spektren ((a) Realteil $\varepsilon ;(b)$ Imaginärteil $\varepsilon^{\prime \prime) ~ b e i ~} 1 \mathrm{GHz}$ in Abhängigkeit von der Temperatur für ein kommerzielles reines Cyanatesterharz (L 10) sowie für zwei Modifizierungen des Harzes mit 1 Ma-\% bzw. 3 Ma-\% Siliziumcarbid (SiC).

\subsubsection{Messtechnische Aspekte}

Durch Kombination verschiedener Messprinzipien ist mit der am Fraunhofer IAP, Forschungsbereich Polymermaterialien und Composite PYCO, zur Verfügung stehenden Messtechnik die dielektrische Funktion $\varepsilon^{*}(f, T)$ in einem Frequenzbereich von $10^{-6} \mathrm{~Hz}$ bis $3 \cdot 10^{9} \mathrm{~Hz}$ sowie in einem Temperaturbereich von $-160^{\circ} \mathrm{C}$ bis $400^{\circ} \mathrm{C}$ zugänglich (Kremer et al. 2002). Zu ihrer Bestimmung werden zwei verschiedene Messsysteme eingesetzt. Im Frequenzbereich bis zu $10 \mathrm{MHz}$ wird die Probe als Dielektrikum in einem Kondensator realisiert, da die Einflüsse und Verluste durch die Zuleitungen nicht ins Gewicht fallen bzw. kompensiert werden können. Die Leerkapazität $C_{0}$ eines Plattenkondensators mit der Fläche $A$ und dem Plattenabstand $d$ ist $C_{0}=\varepsilon_{0} A d d$. Die dielektrische Funktion $\varepsilon^{*}(f)$ erhält man aus der gemessenen komplexen Impedanz $Z^{*}(f)$ des Systems zu $\varepsilon^{*}(f)=\frac{1}{i 2 \pi f C_{0} Z^{*}(f)}$. 


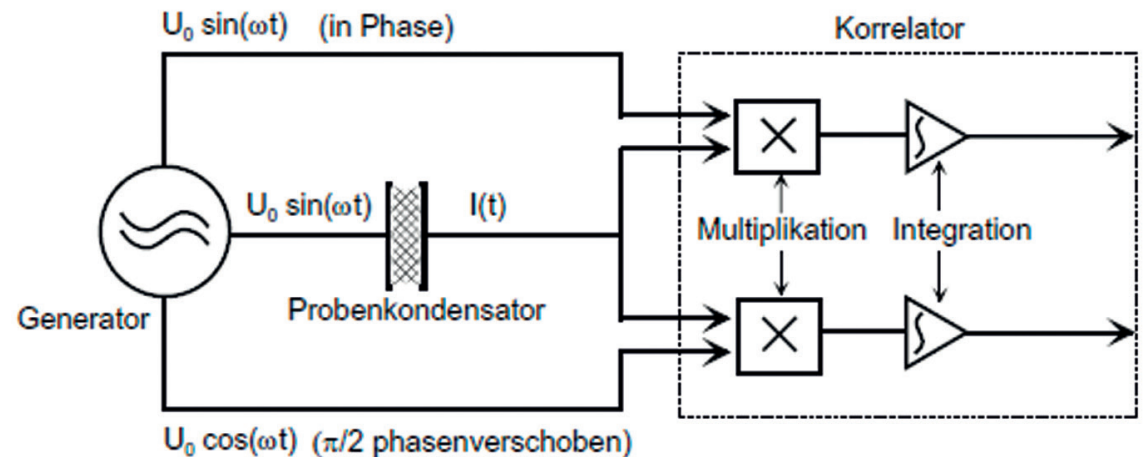

Abb. 3) Funktionsprinzip eines Frequenzganganalysators nach Kremer et al. 2002.

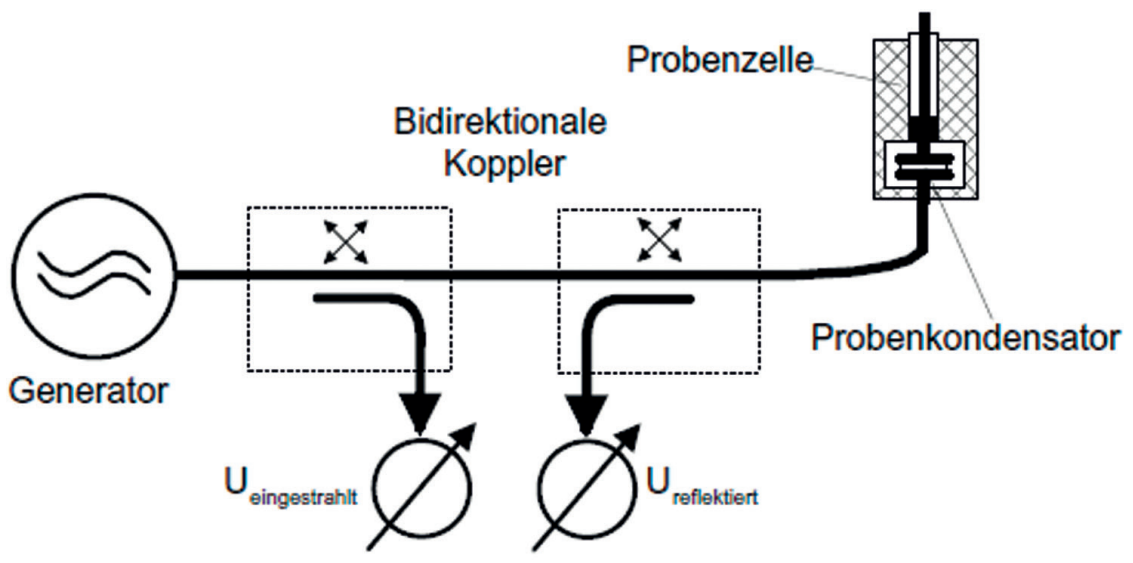

Abb. 4) Funktionsprinzip eines koaxialen Reflektometers nach Kremer et al. 2002.

Im Frequenzbereich bis $10 \mathrm{MHz}$ wird die Impedanz mit Hilfe des in Abb. 3 gezeigten Frequenzganganalysators gemessen. An den Probenkondensator wird eine sinusförmige Spannung angelegt. Der resultierende Strom $I(t)$ wird jeweils mit zwei Signalen korreliert, die in Phase und um $90^{\circ}$ phasenverschoben zur Generatorspannung sind. Nach Integration über eine bestimmte Anzahl von Perioden erhält man die fouriertransformierte Antwort des Systems. Daraus lässt sich die komplexe Impedanz und die dielektrische Funktion errechnen. Die Messungen werden mit einem Alpha-Analyzer der Firma Novocontrol vorgenommen, mit dem Impedanzen von $10^{-1} \Omega$ bis $10^{14} \Omega$ mit einer Auflösung in $\tan \delta=\varepsilon^{\prime} / \varepsilon^{\prime \prime}$ von weniger als $10^{-5}$ im gesamten Frequenzbereich gemessen werden können.

Für den Frequenzbereich von $10^{6} \mathrm{~Hz}$ bis $3 \cdot 10^{9} \mathrm{~Hz}$ wird ein koaxiales Reflektometer des Typs E4991A der Firma Agilent eingesetzt, dessen Funktionsprinzip in Abb. 4 skizziert ist. Die Probe befindet sich am Ende eines koaxialen
Hohlleiters. Am Innenleiter wird die Generatorspannung angelegt. Die Spannungen der eingestrahlten und der reflektierten Welle werden mit zwei bidirektionalen Kopplern gemessen. Der komplexe Reflexionskoeffizient $r^{*}$ ist $r^{*}=U_{\text {reffektiert }} / U_{\text {eingestrahl }}$. Ist die Impedanz $Z_{0}$ des Koaxialleiters bekannt, dann kann die Impedanz $Z^{*}$ der Probe

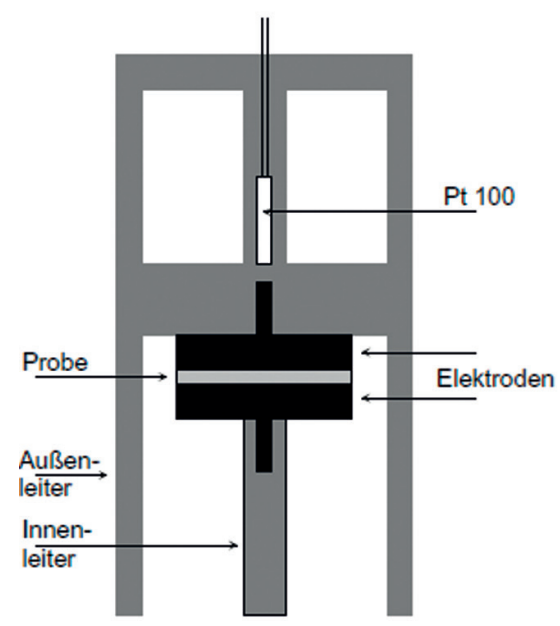

Abb. 5) Probenhalterung des koaxialen Reflektometers nach Kremer et al. 2002. bestimmt werden zu: $Z^{*}=Z_{0} \frac{1+r^{*}}{1-r^{*}}$. Die Auflösung in tan $\delta$ ist im gesamten Bereich besser als $10^{-2}$. Die verwendete Probenzelle ist in Abb. 5 gezeigt.

Die Thermostatisierung der Probe im o. g. Temperaturbereich wird für beide Messsysteme durch eine StickstoffGasstrom-Heizung realisiert. Flüssiger Stickstoff wird verdampft und erwärmt, bevor er den Probenhalter erreicht. Gas- und Probentemperatur werden jeweils mit einem Pt-100-Element gemessen, wobei der Sensor von einer Bohrung im Probenhalter aufgenommen wird. Die Heizung wird von einer Vierkreis-Regeleinheit gesteuert, mit der eine minimale Temperaturdrift von $0,05 \mathrm{~K} \mathrm{~min}^{-1}$ und eine Genauigkeit von $0,05 \mathrm{~K}$ in der absoluten Temperatur erreicht werden können.

\subsection{Numerische Simulation elektromagnetischer Felder in Mikrowellenöfen}

Neben ihrer Verwendung für die Charakterisierung dielektrischer Materialien im Allgemeinen dienen die dielektrischen Kennwerte auch zur Abschätzung (vgl. Abschnitt 2) bzw. zur Simulation der Erwärmung von Materialien in Mikrowellenöfen. Letzteres wird im Folgenden beispielhaft für die Erwärmung eines modifizierten Harzes im Monomode-Mikrowellenofen (vgl. Abschnitt 4.2) demonstriert, wobei für die Simulation der elektromagnetischen Felder die CST Microwave Studio Suite ${ }^{\circledR}$ verwendet wurde. Grundsätzlich erfordern die Simulationen ein geometrisches Modell des jeweiligen Mikrowellenofens samt des darin befindlichen Werkstückes, wobei den einzelnen Komponenten ihre jeweiligen Materialeigenschaften wie Leitfähigkeit oder dielektrische Permittivität zuzuweisen sind. Da es sich bei den Mikrowellenöfen um „elektrisch große“ Objekte handelt mit Abmessungen typischerweise größer als die Wellenlänge des elektromagnetischen Feldes (ca. $12 \mathrm{~cm}$ für eine Mikrowellenfrequenz von 2,45 $\mathrm{GHz}$ ), ist eine vereinfachte geometrische Modellierung ausreichend. Ferner sind die Quellen des elektromagnetischen Feldes (im Falle der Mikrowellenöfen die Magnetrons) in ihrer Leistung und Abstrahlungscharakteristik zu modellieren. Die Simulation der 
elektromagnetischen Felder bedeutet nun, für das jeweilige Modell die Maxwellschen Gleichungen in Integralform sowie die elektromagnetischen Materialgleichungen unter den durch das Modell vorgegebenen Randbedingungen zu lösen. In der o. g. Software ist hierfür die Finite-Integral-Methode implementiert, mittels derer die Lösung des genannten Systems von Differentialgleichungen auf ein algebraisches Gleichungssystem zurückgeführt wird. Hierfür wird das Volumen des gesamten Modells in ein Gitter hinreichend kleiner quaderförmiger Zellen zerlegt. Indem die Maxwellschen Gleichungen unter Beachtung der Stetigkeitsbedingungen für jede Zelle angesetzt werden, lassen sich ihre Integrale durch Produkte ersetzen und es gelingt somit der Übergang zu einem algebraischen Gleichungssystem.
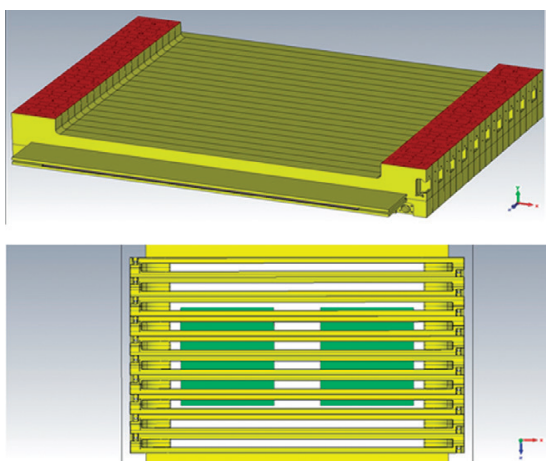

Abb. 6) Gesamtes Modell des Monomode-Mikrowellenofens in der Außenansicht (oben) und in Schnittansicht (unten, die Schnittebene verläuft durch den oberen Teil der Wellenleiter). Die grünen Komponenten sind exemplarische Proben, die im Monomodeofen bestrahlt werden.

In Abb. 6 ist exemplarisch das gesamte Modell des Monomode-Ofens (vgl. Abschnitt 4.2) in der Außenansicht sowie ein Schnitt durch den oberen Teil der Wellenleiter gezeigt. Die grünen Komponenten in Abb. 6 sind exemplarische Proben auf dem Transportband des Ofens.

Für das Zusammenspiel von breitbandiger dielektrischer Spektroskopie und Simulation der elektromagnetischen Felder im Monomode-Ofen sei auf die Abb. 7 bis 9 verwiesen, in denen der Einfluss der Modifizierung eines reinen Cyanatesterharzes (kommerziell erhältliches L 10) mit Mikrowellensuszeptoren (Siliziumcarbid-Körner, Graphen-Flocken und zwei Ruß-Sorten)
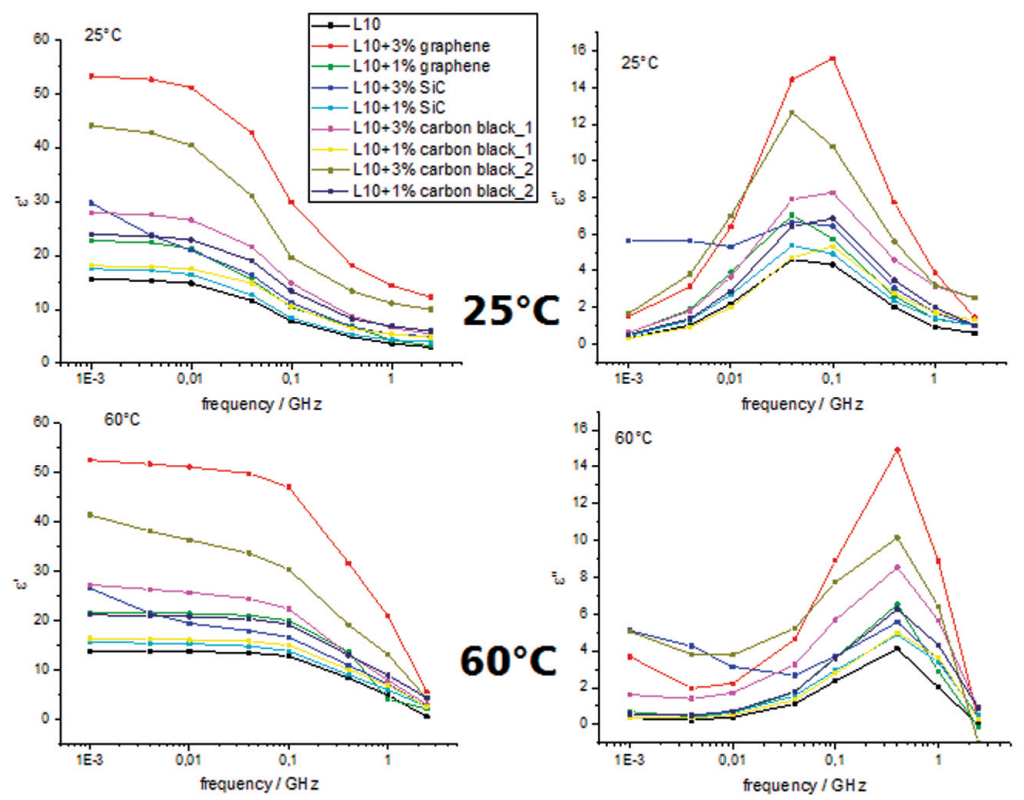

Abb. 7) Dielektrische Spektren des reinen Cyanatesterharzes $L 10$ sowie von Modifizierungen mit Siliziumcarbid, Graphen und zwei Arten Ruß bei $25^{\circ} \mathrm{C}$ und $60^{\circ} \mathrm{C}$.

auf das Eindringen des elektrischen Feldes in eine „Harz-Platte“ sowie auf die resultierende Leistungsverlustdichte untersucht wird. Die Abmessungen der Probe sind so gewählt, dass sie den Innenraum des Monomode-Ofens ausfüllen.

Zunächst zeigt Abb. 7, dass bereits im Falle des Reinharzes für Temperaturen zwischen $25^{\circ} \mathrm{C}$ und $60{ }^{\circ} \mathrm{C}$ der Frequenzbereich zwischen $1 \mathrm{MHz}$ und $3 \mathrm{GHz}$ bestimmt ist durch einen molekularen Relaxationsprozess, dessen Maximumslage in $\varepsilon$ " sich mit steigender Temperatur zu höheren Frequenzen verschiebt. Allein aufgrund dieser intrinsischen Frequenz- und Temperaturabhängigkeit der dielektrischen Kennwerte zeigt sich das dielektrische Heizen als ein sich selbst verstärkender (oder u. U. auch abschwächender) Prozess: Die Absorption der Mikrowellen in der Probe bewirkt eine initiale Erwärmung, infolge derer sich die dielektrischen Kennwerte ändern, was wiederum zu einer veränderten Leistungsverlustdichte bzw. Eindringtiefe führt. Die Mikrowellensuszeptoren bewirken eine weitere Erhöhung der dielektrischen Kennwerte, deren Ausmaß vom jeweiligen Suszeptor abhängt. Die Daten in Abb. 7 wurden direkt für die elektromagnetischen Simulationen verwendet, indem ein Debye-Relaxationsprozess angenommen und die
Daten auf Frequenzen von $2,45 \mathrm{GHz}$ extrapoliert wurden.

Abb. 8 zeigt exemplarisch für Temperaturen von $25^{\circ} \mathrm{C}$ und $60{ }^{\circ} \mathrm{C}$ die Simulationsergebnisse für die elektrische Feldstärke $\boldsymbol{E}$ und die Leistungsverlustdichte in der Probe des reinen Cyanatesterharzes $L$ 10. Die Ergebnisse sind in einer Ebene bei der halben Probendicke gezeigt; für die Darstellung der Ergebnisse beider Temperaturen wurde jeweils die gleiche Skala verwendet. Bei Raumtemperatur sind die elektrische Feldstärke und die Leistungsverlustdichte durch stehende Wellen bestimmt, die aus den Wellenleitern des MonomodeOfens direkt in die Probe einkoppeln. Bei $60{ }^{\circ} \mathrm{C}$ führt die Zunahme der dielektrischen Kennwerte offensichtlich zu einer verringerten Eindringtiefe des elektrischen Feldes in das Innere der Harzplatte und folglich auch zu einer Reduktion der Leistungsverlustdichte.

Abb. 9 zeigt für Temperaturen von $25^{\circ} \mathrm{C}$ und $60^{\circ} \mathrm{C}$ die simulierte Leistungsverlustdichte im reinen Cyanatesterharz L 10 sowie in Formulierungen mit jeweils 3 Ma-\% Siliziumcarbid bzw. Graphen. Die markantesten Ergebnisse sind zum einen der Abfall der Leistungsverlustdichte im Inneren der Probe für die Graphen-Formulierung bei $60{ }^{\circ} \mathrm{C}$ gegenüber dem reinen Harz sowie zum anderen der moderate 

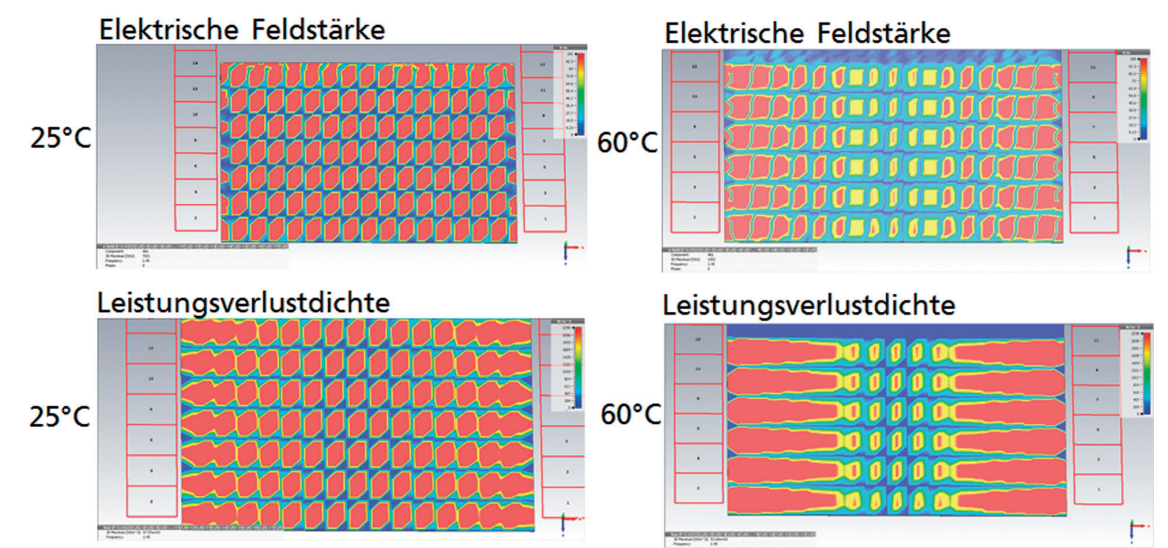

Abb. 8) Simulationsergebnisse der elektrischen Feldstärke (obere Zeile) und der Leistungsverlustdichte (untere Zeile) des reinen Cyanatesterharzes $L 10$ bei $25^{\circ} \mathrm{C}$ (linke Spalte) und $60^{\circ} \mathrm{C}$ (rechte Spalte).

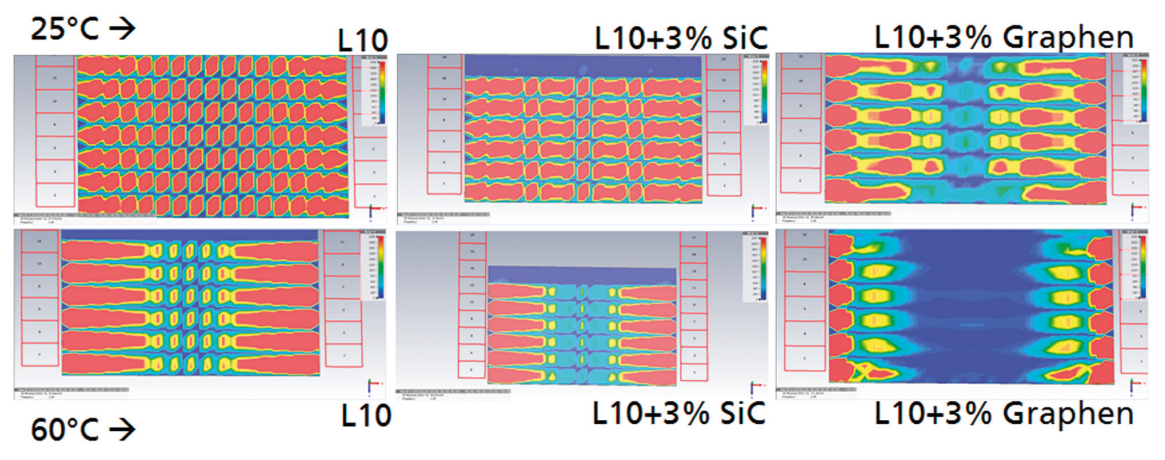

Abb. 9) Leistungsverlustdichte des reinen und modifizierten Cyanatesterharzes $L 10$ bei $25^{\circ} \mathrm{C}$ (obere Zeile) und $60^{\circ} \mathrm{C}$ (untere Zeile).

Anstieg der Leistungsverlustdichte bei $25{ }^{\circ} \mathrm{C}$ für die mit Siliziumcarbid modifizierte Probe. Indem sie den komplexen Zusammenhang zwischen einer Modifizierung des Harzes und der in ihm auftretenden Leistungsverlustdichte bei Mikrowellenbestrahlung aufzeigen, bestätigen die elektromagnetischen Simulationen im Zusammenspiel mit der breitbandigen dielektrischen Spektroskopie ihren Wert für Untersuchungen zur Erwärmung von Materialien mittels Mikrowellenbestrahlung.

\section{Mikrowellentechnik - Kammeröfen und Banddurchlauföfen}

Die in der Forschungsgruppe Thermosets im Leichtbau im Rahmen der Kooperation zwischen der TH Wildau und dem Fraunhofer IAP, Forschungsbereich Polymermaterialien und Composite PYCO, zur Verfügung stehende Mikrowellentechnik umfasst sowohl einen Mikrowellenkammerofen vom Typ Hephaistos als auch zwei eigens entwickelte Banddurchlauföfen, einen Multimodeund einen Monomode-Applikator, die

im Folgenden eingehender vorgestellt werden. Grundsätzlich ist damit sowohl die Mikrowellenbehandlung großer Bauteile mit Abmessungen im Metermaßstab im Batch-Betrieb als auch dünner Materialien wie Folien, Prepregs und Platten sowie kleiner Bauteilen in einem kontinuierlichen Betrieb möglich.
4.1 Mikrowellen-Kammerofen Hephaistos

Abb. 10 zeigt einen Mikrowellenkammerofen vom Typ Hephaistos des Herstellers Vötsch, der auf umfangreichen Vorentwicklungen am Karlsruher Institut für Technologie (KIT) beruht (Feher $\&$ Thumm 2004). Durch den hexagonalen Kammerquerschnitt wird ein homogenes elektromagnetisches Feld in der Probenkammer erreicht. Die Tiefe der Probenkammer beträgt $3 \mathrm{~m}$ und der Durchmesser des Hexagon-Umkreises $1,8 \mathrm{~m}$, woraus sich ein maximales Volumen zu behandelnder Proben von ca. $8 \mathrm{~m}^{3}$ ergibt. Entlang der sechs Seitenflächen des die Kammer begrenzenden Hexagons sind insgesamt $36 \mathrm{Ma}$ gnetrons als Quellen der Mikrowellen platziert (pro Seitenfläche gibt es zwei Reihen mit je drei hintereinander platzierten Magnetrons). Über Schlitzantennen in den außen an den Seitenflächen des Hexagons angebrachten Wellenleitern werden die Mikrowellen in das Innere des Hexagons eingekoppelt. Alle Magnetrons erzeugen elektromagnetische Wellen der Frequenz $2,45 \mathrm{GHz}$ mit einer Leistung von jeweils $0,85 \mathrm{~kW}$.

Die Messung der Materialtemperatur erfolgt in Kontakt mit dem Material über 12 faseroptische Sensoren der Firma Optocon, denen die Temperaturabhängigkeit der Lage der Bandkante eines Halbleiters zugrunde liegt. Das Messprinzip ist insbesondere unempfindlich gegen elektromagnetische

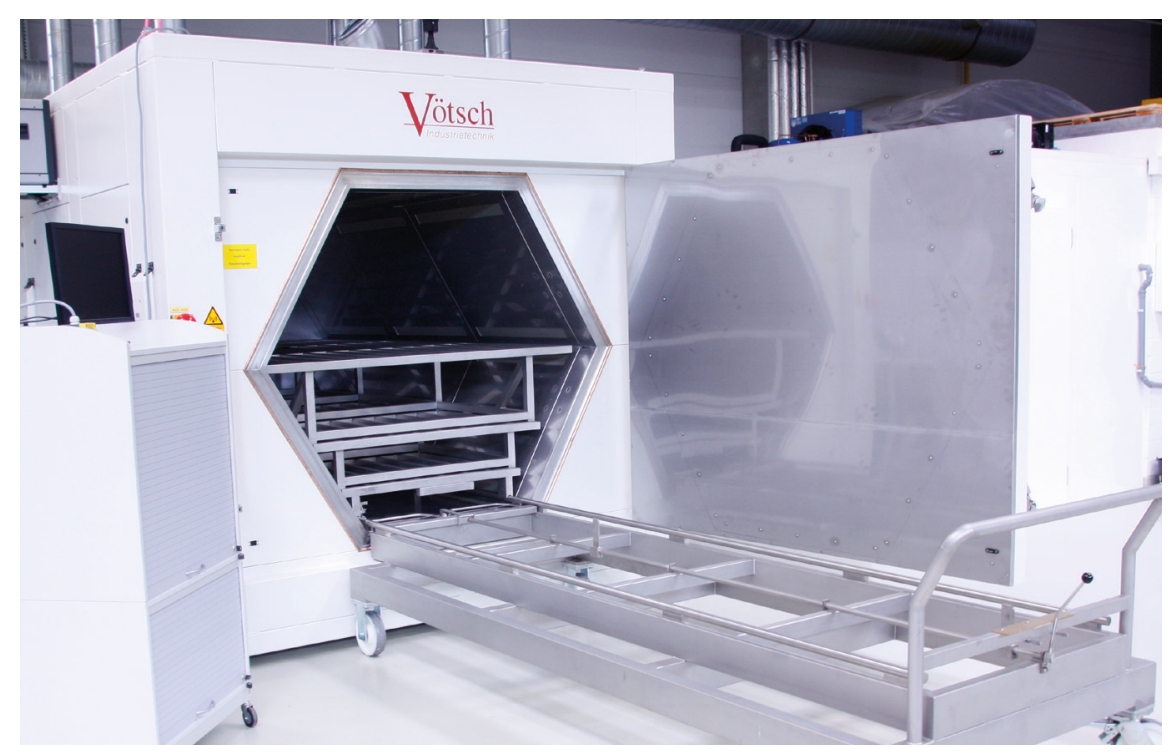

Abb. 10) Mikrowellenkammerofen Hephaistos des Herstellers Vötsch. 
Felder in der Einsatzumgebung und die Sensoren leiten die Felder auch nicht aus dem Mikrowellenofen heraus. Die Leistung aller aktivierten Magnetrons kann durch einen PID-Regler so geregelt werden, dass sich für einen beliebig auswählbaren faseroptischen Sensor ein vorgegebener Sollwert einstellt. Für eine Bewertung der Temperaturverteilung auf der Materialoberfläche ist in der Decke des Hexagons eine Infrarot-Kamera installiert.

\subsection{Mikrowellen-Banddurchlauföfen: Monomode- und Multimode- Applikator}

Abb. 11 zeigt den aus einem Multimode- und einem Monomode-Applikator bestehenden Mikrowellen-Banddurchlaufofen, wie er als Versuchsanlage zur Mikrowellenbestrahlung flacher Materialien in einem kontinuierlichen Betrieb der Forschungsgruppe am Fraunhofer IAP zur Verfügung steht (Dreyer et al. 2015). Im Momomode-Applikator werden die zu bestrahlenden Proben auf einem glasfaserverstärkten TeflonTransportband direkt durch die seitlich geschlitzten, insgesamt sechs Wellenleiter geführt, in die jeweils ein Magnetron mit einer Frequenz von $2,45 \mathrm{GHz}$ und einer maximalen Leistung von 1,3 kW einkoppelt. Diese Bauform ermöglicht hohe Feldstärken bei der 1. Harmonischen der eingekoppelten Mikrowellen, begrenzt jedoch die Dicke zu bestrahlender Proben auf $8 \mathrm{~mm}$.

Im Multimode-Applikator werden die in den Magnetrons generierten Mikrowellen analog zum Hephaistos-Kammerofen über Schlitzantennen aus außen angebrachten Wellenleitern in das Innere der Kammer eingekoppelt. In diesem Fall sind Dicken des zu bestrahlenden Materials von bis zu ca. $80 \mathrm{~mm}$ möglich. Es sind ober- und unterhalb der Kammer jeweils neun Magnetrons angebracht, von denen 16 bei $2,45 \mathrm{GHz}$ laufen und eine Leistung von maximal 1,3 kW bereitstellen. Zwei Magnetrons laufen hingegen bei $5,8 \mathrm{GHz}$ mit einer Leistung von maximal $0,8 \mathrm{~kW}$. Bezogen auf die Standardfrequenz von 2,45 GHz erlaubt die Verwendung der ca. doppelt so großen Frequenz eine Bestrahlung, die bei sonst gleichen Bedingungen gegenüber $2,45 \mathrm{GHz}$ eine verminderte Eindringtiefe und eine

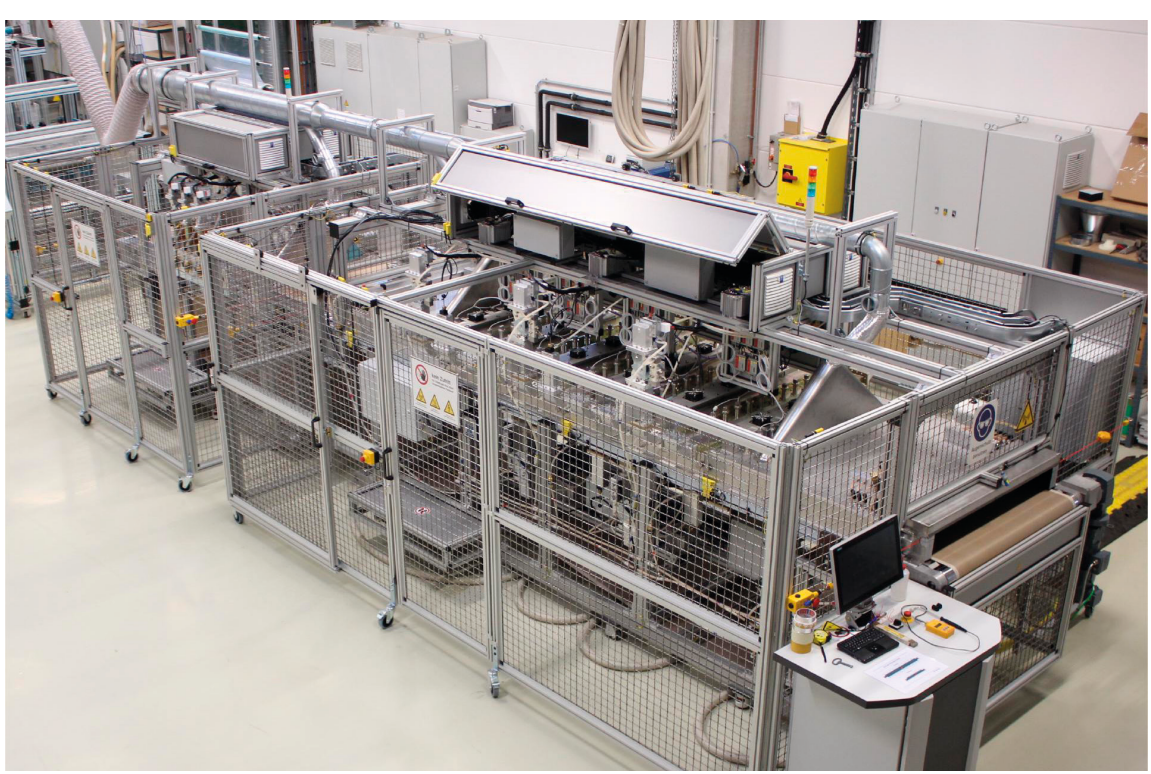

Abb. 11) Zweigeteilter Mikrowellen-Banddurchlaufofen des Fraunhofer IAP bestehend aus einem MonomodeApplikator (hinten) und einem Multimode-Applikator (vorn).

Erhöhung der Leistungsverlustdichte gemäß Abschnitt 2.1 aufweist. Zusätzlich zu der beschriebenen Mikrowelleneinheit ist der Multimode-Applikator mit insgesamt 6 Infrarot-Strahlern einer Leistung von jeweils $6 \mathrm{~kW}$ bestückt, die neben der reinen Bestrahlung mittels Mikrowellen auch einen klassischen Ofenbetrieb bzw. einen kombinierten Betrieb zulassen. Analog zum Monomode-Applikator erfolgt der Transport des zu bestrahlenden Materials mittels eines glasfaserverstärkten Teflon-Transportbandes, das im Langzeitbetrieb mit Temperaturen von bis $\mathrm{zu} 200^{\circ} \mathrm{C}$ belastbar ist.

Die Messung der Materialtemperatur in Kontakt kann in beiden Applikatoren mittels der oben beschriebenen faseroptischen Sensoren erfolgen. Darüber hinaus wird die Temperatur im Inneren beider Kammern kontaktlos über eine Matrix von Pyrometern überwacht, die jeweils in der Oberseite der Kammern angebracht sind. Im Unterschied zum Hephaistos-Ofen ist bei beiden Banddurchlauföfen derzeit nur ein ungeregelter Betrieb möglich.
5. Beispiele zur Mikrowellenbehandlung von Materialien

Im Folgenden werden drei Beispiele für die thermische Behandlung unterschiedlicher Materialien mittels Mikrowellen vorgestellt, wobei jeweils einer der zuvor vorgestellten Mikrowellenöfen mit seinen spezifischen Eigenschaften zum Einsatz kommt. Die Anwendungsfälle umfassen das Tempern, das Trocknen sowie die Initiierung einer chemischen Reaktion mittels Mikrowellen.

\subsection{Einsatz des Monomode-Applikators für die Herstellung von Prepregs}

Es wurde untersucht, inwieweit ein mit einem Epoxidharz imprägniertes Carbonfasergewebe nach kurzer konventioneller Vorhärtung in dem Monomode-Applikator auf Temperaturen von $150{ }^{\circ} \mathrm{C}$ bis $200{ }^{\circ} \mathrm{C}$ gebracht werden kann, die für die Prepreg-Herstellung relevant sind. Bei Prepregs (von preimpregnated fibers) handelt es sich um Halbzeuge faserverstärkter Kunststoffe, bei denen die Harzmatrix durch teilweises Vorhärten mechanisch stabilisiert ist, die endgültige Formgebung und Härtung jedoch abschließend während eines Produktionsprozesses erfolgen. Gelingt die angestrebte Temperierung des imprägnierten Gewebes, so eröffnet sich die Möglichkeit, die horizontale Imprägnieranlage des Fraunhofer IAP mit der Mikrowelle zu kombinieren: 


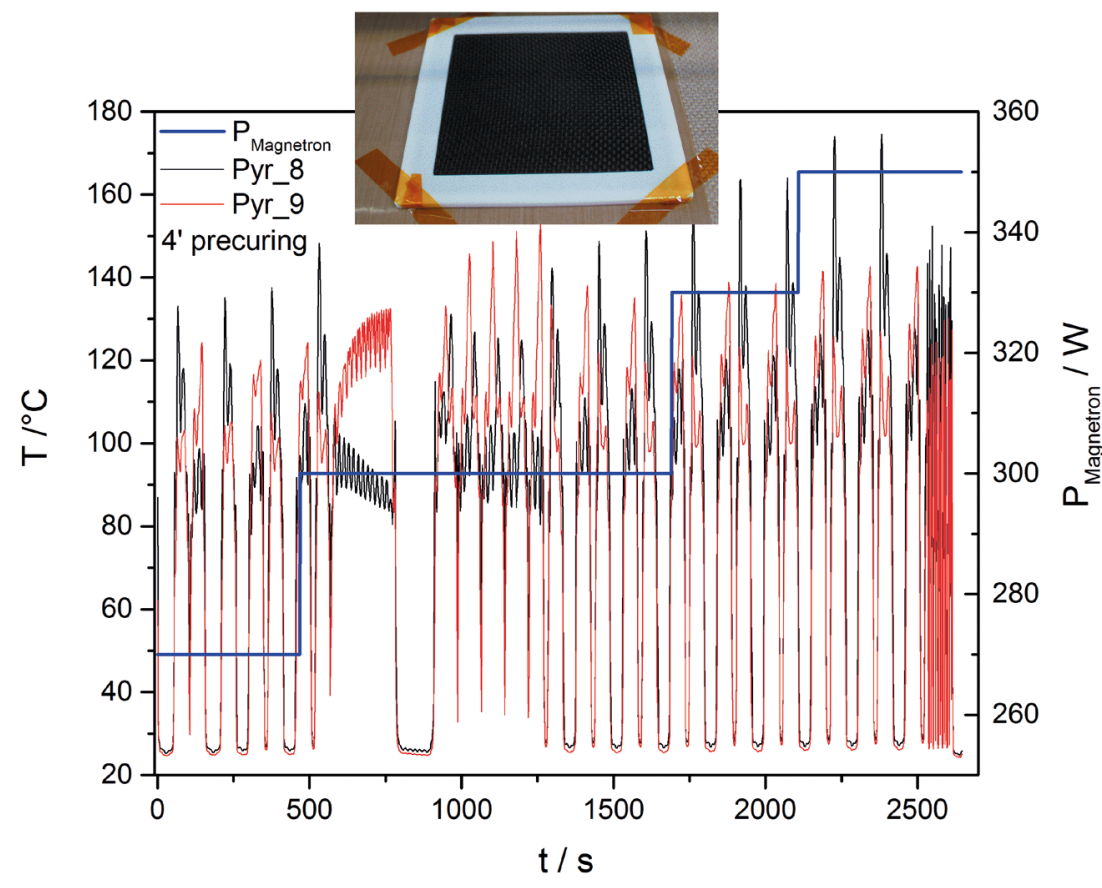

Abb. 12) Zeitlicher Verlauf von Magnetron-Leistung und Probentemperatur eines Epoxidharz-imprägnierten Kohlefasergewebes (Foto) während der Bestrahlung im Monomode-Applikator.

Die herkömmliche Herstellung der Prepregs, basierend auf Kontakt- oder Umlufttrocknern zur thermischen Behandlung der Materialien, kann ersetzt werden durch einen mikrowellen-basierten Prozess, der gegenüber dem konventionellen Herstellungsprozess schneller ist und einen geringeren Energieeinsatz erfordert.

Abb. 12 zeigt den zeitlichen Verlauf der Leistung aller Magnetrons des Monomode-Applikators sowie der aus der Bestrahlung resultierenden Probentemperatur. Diese wurde mit zwei Pyrometern oberhalb der Probe gemessen, unter denen die auf dem Transportband fixierte Probe während der Bestrahlung hin und her bewegt wurde. Das Foto in Abb. 12 vermittelt einen Eindruck der Probe: Für den hier vorgestellten Versuch wurde ein Stück Carbonfasergewebe einer Fläche von ca. $15 \mathrm{~cm} \times 15 \mathrm{~cm}$ gewählt, mit einem Epoxidharz imprägniert und anschließend bei $160{ }^{\circ} \mathrm{C}$ für $4 \mathrm{~min}$ in einem Trockenschrank vorvernetzt. Um die aus Spitzenentladungen an den Kohlefaserenden resultierende Funkenbildung zu unterbinden, wurden die Ränder der Probe mit einem TeflonRahmen ummantelt. In den ersten Versuchen wurde die Leistung sukzessive erhöht, um einen zu heftigen Reaktionsverlauf der Harzmatrix zu vermei- den. Mittels dieser Prozessführung wurde mit einer einheitlichen Leistung aller Magnetrons von $350 \mathrm{~W}$ eine stabile Endtemperatur der Probe von ca. $160{ }^{\circ} \mathrm{C}$ erreicht, was für eine spätere Prepreg-Herstellung für die meisten verwendeten Harzsysteme ausreichend ist, zumal hinsichtlich der MagnetronLeistung noch Spielraum für eine weitere Temperaturerhöhung besteht.

\subsection{Trocknung von Dichtungsmateria- lien im Multimode-Applikator}

Strang-Materialien für Hochtemperatur-Dichtungen mit einem Anwendungsbereich von $800{ }^{\circ} \mathrm{C}$ bis $900{ }^{\circ} \mathrm{C}$ werden hergestellt, indem diese während der Herstellung mit wässrigen Dispersionen getränkt und anschließend getrocknet werden. Diese Trocknung erfolgt bei $30{ }^{\circ} \mathrm{C}$ über mehrere Tage hinweg. Im vorliegenden Anwendungsbeispiel wurde untersucht, inwieweit dieser Trocknungsprozess durch den Einsatz des Multimode-Applikators des Banddurchlaufofens signifikant beschleunigt werden kann.

Wie in Abb. 13 gezeigt, wurden hierzu Abschnitte des unbehandelten Strang-Materials (Referenz) wie auch verschieden getränkte Abschnitte auf dem Transportband des MultimodeApplikators fixiert und anschließend

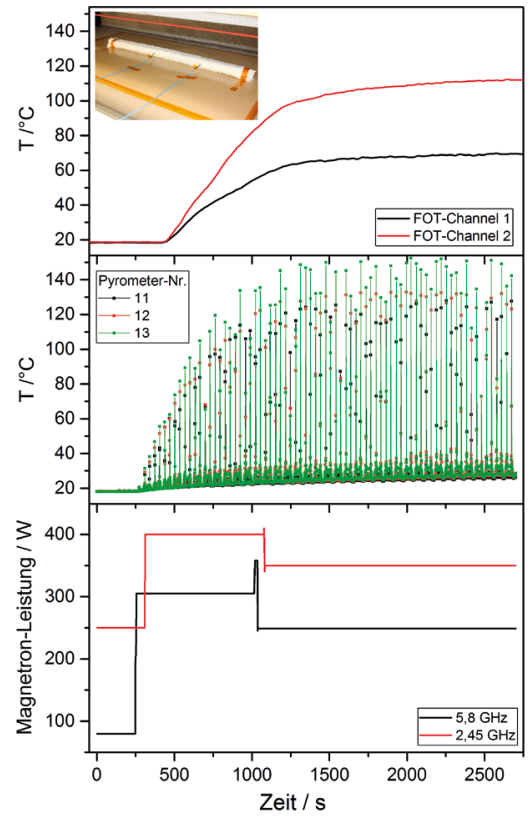

Fig. 13) Zeitlicher Verlauf von Magnetron-Leistung (unten) und Probentemperatur (Mitte, oben) eines Strangmaterials für Hochtemperaturdichtungen (hier: Referenz, s. Foto) während seiner Trocknung durch Bestrahlung im Multimode-Applikator. Die Temperaturmessung erfolgte sowohl durch faseroptische Sensoren (FOT, oben) als auch mittels der integrierten Pyrometer (Mitte).

unter Verwendung des Oszillationsmodus des Transportbandes im Multimode-Applikator bestrahlt. Die Temperaturmessungen erfolgten sowohl mittels der Pyrometer (Messung der Oberflächentemperatur) als auch mittels der faseroptischen Sensoren, die so fixiert wurden, dass ihre Messspitzen die Temperatur im Inneren des Strangmaterials erfassen.

Abb. 13 zeigt exemplarisch für die Referenz, wie sich infolge der simultanen Bestrahlung mit beiden zur Verfügung stehenden Frequenzen bei Leistungen im unteren Bereich nach ca. 15 min eine Oberflächentemperatur von ca. $120^{\circ} \mathrm{C}$ einstellt, während sich das Stranginnere dem gegenüber verzögert auf niedrigere Temperaturen zwischen $60^{\circ} \mathrm{C}$ und $100{ }^{\circ} \mathrm{C}$ erwärmt. Die Ursache für die inhomogene Erwärmung ist noch nicht verstanden, allerdings stimmt der Abschnitt des Strangmaterials mit der höchsten gemessenen Oberflächentemperatur (bis $140{ }^{\circ} \mathrm{C}$ durch Pyrometer 13) mit dem der höchsten Temperatur im Stranginneren (bis $100{ }^{\circ} \mathrm{C}$ durch den faseroptischen Sensor FOT 2) überein.

Im Ergebnis zeigte sich für eine solcherart durchgeführte mikrowellenbasierte 
Trocknung über jeweils ca. $1 \mathrm{~h}$ für das Referenzmaterial erwartungsgemäß ein vernachlässigbarer Masseverlust von nur 1 bis 2 Ma-\%, während für unterschiedlich getränkte Stränge in der gleichen Zeit ein Massenverlust zwischen $28 \mathrm{Ma}$ \% und $35 \mathrm{Ma} \%$ erreicht wurde. Ungeachtet des noch ausstehenden Vergleiches mit den entsprechenden Werten der herkömmlichen Trocknung zeichnet sich bereits ab, dass die mikrowellenbasierte Trocknung deutlich schneller und damit auch energiesparender ablaufen würde als der herkömmliche Trocknungsprozess.

\subsection{Tempern eines harzgebundenen Reibbelags im Hephaistos-Ofen}

Bei der Herstellung von harzgebundenen Reibbelägen für unterschiedliche Anwendungen ist ein essentieller Prozessschritt ein der eigentlichen Herstellung nachgelagertes Tempern bei Temperaturen zwischen $150{ }^{\circ} \mathrm{C}$ und $200^{\circ} \mathrm{C}$, um das endgültige Eigenschaftsprofil der Beläge einzustellen.

Abb. 14 zeigt die Erwärmung eines harzgebundenen Reibbelags, dessen Vorbereitung für das Tempern im eingesetzten Foto der Abbildung dargestellt ist: Auf der konkaven Seite

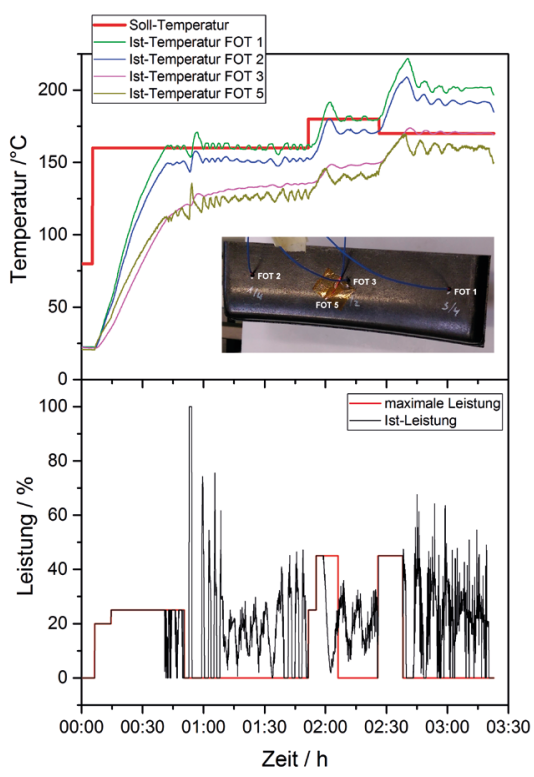

Fig. 14) Zeitlicher Verlauf von Magnetron-Leistung (unten) und Probentemperatur (oben, gemessen mit faseroptischen Sensoren, s. Foto) für das NachTempern eines harzgebundenen Reibbelags im Hephaistos-Mikrowellenofen. Der Ofen wird dabei im geregelten Modus betrieben. des Reibbelags wurden insgesamt drei Bohrungen angebracht. Jede der Bohrungen wurde mit einem faseroptischen Temperatursensor (FOT) nach folgendem Schema ausgestattet:

- FOT 1: rechte Bohrung bis $3 / 4$ der Belagdicke;

- FOT 2: linke Bohrung bis $1 \frac{1}{4}$ der Belagdicke;

- FOT 3: mittlere Bohrung bis $1 / 2$ der Belagdicke

- FOT 5: Anbringung mittig an der konkaven Belagoberfläche

Der Belag wurde anschließend im Zentrum des in drei Segmente unterteilten Hephaistos-Ofens auf dem zugehörigen Probenwagen auf einer Teflon-Platte platziert, wobei nur die 12 Magnetrons des mittleren Segments aktiviert wurden. In einem ersten Abschnitt (bis ca. 1 h 50 min ab Beginn des Bestrahlens) wurde die Regelung auf eine Solltemperatur von $160^{\circ} \mathrm{C}$ für FOT 1 untersucht. Diese wird nach ca. 35 min erreicht, wobei zu beachten ist, dass die Leistung der Mikrowelle (d.h. der 12 verwendeten mittleren Magnetrons) zunächst auf $25 \%$ der Maximalleistung begrenzt wurde."

Es bleibt daher noch zu untersuchen, inwieweit sich mit höheren MagnetronLeistungen bzw. durch Einbeziehung weiterer Magnetrons kürzere Heizzeiten erzielen lassen. Nach dem Ende des ersten Abschnittes wurde für einen zweiten Abschnitt (bis ca. 2 h 25 min) die Solltemperatur für FOT 1 auf $180{ }^{\circ} \mathrm{C}$ gesetzt; für das Erwärmen um weitere $20 \mathrm{~K}$ werden etwa $15 \mathrm{~min}$ benötigt, bis die Regelung greift. Bereits nach 7 min wird der Sollwert erstmals erreicht. In einem dritten Abschnitt ( $a b$ 2 h 25 min) wird die Solltemperatur auf $170{ }^{\circ} \mathrm{C}$ für FOT $3 \mathrm{im}$ Zentrum des Belages gesetzt. Die Solltemperatur wird nach ca. 12 min erreicht, wobei auffällt, dass die Regelung, vermutlich aufgrund der größeren thermischen Masse, besser „greift", d. h. es gibt keine Oszillation der Ist-Temperatur um den Sollwert. Die in Abb. 14 gezeigten Daten geben auch einen Einblick in die Temperaturverteilung innerhalb des Belags während der Erwärmung mittels Mikrowellen. Im Gegensatz zu einem herkömmlichen Ofen bleibt der Belag an der Oberfläche am kältesten, während die stärkste Erwärmung für die beiden Enden des Belags registriert wird und das Zentrum des Belags eine Mittelstellung zwischen diesen Extremen einnimmt. Während das Tempern von Reibbelägen im Hephaistos-Ofen hinsichtlich der erreichten Temperatur gelingt, ist eine Verbesserung der Homogenität der Materialtemperatur wünschenswert. Hierfür soll eine periodische Bewegung des Belags während der Bestrahlung untersucht werden, wofür der Multimode-Durchlaufofen eingesetzt werden soll. Dahinter steht die Annahme, dass es durch Mittelung über das in der Ofenkammer variierende elektrische Feld letztlich zu einer homogeneren Erwärmung der Reibbeläge kommt.

\section{Zusammenfassung und Ausblick}

Im vorliegenden Beitrag werden sowohl grundlegende Aspekte der Erwärmung von Materialien durch Bestrahlung mit Mikrowellen erläutert, als auch konkrete Anwendungsbeispiele diskutiert. Hinsichtlich der Grundlagen werden zunächst das dielektrische Heizen als auch das Wirbelstrom-Heizen als die beiden Mechanismen beschrieben, die für die Wandlung elektromagnetischer Feldenergie in Wärme verantwortlich sind. Dabei wird deutlich, dass für eine gegebene Konfiguration eines Mikrowellenofens die Effizienz des Heizens mittels Mikrowellen abhängig ist von der dielektrischen Funktion und der Leitfähigkeit des zu erwärmenden Materials. Beide Materialparameter sind mittels der im Beitrag beschriebenen breitbandigen dielektrischen Spektroskopie in einem ausreichend weiten Temperatur- und Frequenzbereich zugänglich und können insbesondere auch für die Modellierung und Simulation der elektromagnetischen Felder und der Leistungsverlustdichte in Materialien in einem Mikrowellenofen herangezogen werden. Mit derartigen Simulationen lassen sich zum einen Feldüberhöhungen und daraus resultierende Bereiche übermäßig starker Erwärmung (hot spots) vorhersagen. Darüber hinaus können Konzepte für Materialmodifizierungen mit dem Ziel einer effizienteren 
Mikrowellenerwärmung relativ schnell in ihrer Wirkung getestet werden. Im konkreten Fall wurde die Modifizierung eines Cyanatesterharzes mit verschiedenen kohlenstoffbasierten Partikeln untersucht und gezeigt, wie sich diese Partikel auf die Eindringtiefe des elektrischen Feldes und auf die Leistungsverlustdichte auswirken. Im zweiten Teil des Beitrages wurde neben einer Beschreibung der der Forschungsgruppe Thermosets im Leichtbau im Rahmen der $\mathrm{FH}$-Kooperation zwischen der $\mathrm{TH}$ Wildau und dem Fraunhofer IAP, Forschungsbereich PYCO, zur Verfügung stehenden Mikrowellentechnik an dre Beispielen gezeigt, wie für unterschiedliche Anwendungen die dafür erforderlichen Materialien (Carbonfaser-Prepreg, Dichtungsmaterial und Reibbelag) mittels der verschiedenen MikrowellenÖfen thermisch prozessiert werden können.

Ein Schwerpunkt künftiger Arbeiten wird es sein, die mikrowellenbasierte Herstellung von Prepregs auf Glasfasergewebe auszudehnen. Da die Glasfasern selbst als „Wärmesenke“ wirken, muss die Erwärmung der Harzkomponente effizienter gestaltet werden. Hierzu soll vor allem die im Beitrag beschriebene Harzmodifizierung mittels Mikrowellensuszeptoren auf ihre Eignung hin untersucht werden.

\section{Danksagung}

Die vorgestellten Arbeiten wurden im Rahmen des Kooperationsprogrammes zwischen der Fraunhofer-Gesellschaft und der TH Wildau gefördert und in der Forschungsgruppe Thermosets im Leichtbau am Fraunhofer-Institut für Angewandte Polymerforschung IAP, Forschungsbereich Polymermaterialien und Composite gemeinsam mit der Professur Faserverbund-Materialtechnologien der TH Wildau durchgeführt.

\section{LITERATUR}

Akhtar MJ, Feher LE, Thumm MK (2009) Effects of Hardeners, Catalysts and Accelerators on Dielectric Properties of Different Resin Systems for Microwave Material Processing Applications. In: Feher LE (eds), 7.-10. September 2009, Karlsruhe, Germany. Proc. 12th Int. Conf. on Microwave and High Frequency Heating (AMPERE).

Dreyer C, Bauer M, Söthje D, Fischer R (2015) Anordnung zu Behandlung von Materialien mit Mikrowellen. Patent DE102015111555B3, 29 Sep 2016

Feher LE (2009) Energy Efficient Microwave Systems. Springer, Berlin Heidelberg. ISBN: 978-3-540-92121-9

Feher LE, Thumm MK (2004) Microwave innovation for industrial composite fabrication - The HEPHAISTOS technology. IEEE Transactions on Plasma Science 32(1):73-79. doi: $10.1109 /$ TPS.2004.823983

Imenokhoyev I, Windsheimer $\mathrm{H}$, Waitz $\mathrm{R}$, Kintsel $\mathrm{N}$, Linn H (2012) Mikrowellenerwärmungstechnik: Potentiale und Grenzen. cfi/Ber. DKG 89(11-12):D 19-D 27. ISSN: 01739913

Kremer F, Schönhals A (2002) Broadband Dielectric Spectroscopy. Springer-Verlag, Berlin Heidelberg. ISBN: 3-540-43407-0

Link G (2011) Innovative, modulare Mikrowellentechnologie zur Herstellung von Faserverbundstrukturen. Technische Informationsbibliothek u. Universitätsbibliothek, Karlsruhe. doi: 10.2314/GBV:717448614

Link G (2015) Faserverbund-Leichtbau mit Automatisierte Mikrowellenprozesstechnik hoher Energieeffizienz (FLA ME). KIT Scientific Publishing, Karlsruhe. doi: $10.5445 / K S P / 1000047509$

Meredith R (2007) Engineer's Handbook of Industrial Microwave Heating. IET Power Series 25, Exeter. ISBN: 978-0-85296-916-8

Perelaer I, de Gans B-J, Schubert US (2006) Ink-jet printing and microwave sintering of conductive silver tracks. Adv. Mat. 18(16):2101-2104. doi: 10.1002/adma.200502422

Perelaer J, Klokkenburg M, Hendriks CE, Schubert US (2009) Microwave Flash Sintering of Inkjet-Printed Silver Tracks on Polymer Substrates. Adv. Mat. 21(47):48304834. doi: 10.1002/adma.200901081

Zhang H, Yang R, He Y, Foudazi A, Cheng L, Tian G (2017) A review of microwave thermography nondestructive testing and evaluation. Sensors 17(5):1-33. doi: 10.3390/ s17051123

\section{AUTOREN}

Dr. Lutz Hartmann

Tiberiu-Mihai Tatai

Mario Kaiser

Prof. Dr. Christian Dreyer*

Forschungsbereich Polymermaterialen

und Composite PYCO

Forschungsgruppe Thermosets im Leichtbau

Fraunhofer Institut für Angewandte Polymerforschung IAP

* Professur Faserverbund-Materialtechnologien

Technische Hochschule Wildau

denz:

christian.dreyer@th-wildau.de

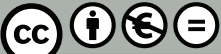

